\title{
Agronomic Practices Influence the Infection of an Oats Cultivar with Fusarium langsethiae
}

\author{
S. M. IMATHIU ${ }^{1}$, R. V. RAY ${ }^{3}$, M. BACK ${ }^{2}$, M. C. HARE ${ }^{2}$ and S. G. EDWARDS ${ }^{2}$ \\ ${ }^{1}$ Faculty of Agriculture, Department of Food Science and Technology, Jomo Kenyatta University of Agriculture \\ and Technology, P.O. Box 62000-00200 Nairobi, Kenya \\ ${ }^{2}$ Harper Adams University, Crop and Environment Sciences, TF10 8NB, UK \\ ${ }^{3}$ School of Biosciences, University of Nottingham, LE12 5RD, UK
}

(Received: 5 November 2016; accepted: 28 November 2016)

\begin{abstract}
Fusarium langsethiae, a toxigenic fungus known to contaminate small-grain cereals with type A trichothecene mycotoxins, HT-2 and T-2 was described as a new species in 2004. HT-2 and T-2 are some of the most potent Fusarium toxins in eukaryotes, capable of inhibiting protein synthesis. The epidemiology of F. langsethiae is not well understood and with the intent of the European Commission to set maximum levels of contamination of cereals with these toxins, importance is currently placed in trying to understand the fungal infection process and its favorable growth conditions. A field study was carried out to investigate the effect of artificially inoculated oats straw, ploughing and minimum tillage with and without incorporated crop debris (straw) on infection and mycotoxin production by $F$. langsethiae on oats cultivar Gerald. The results indicated that cultural field practices had effects on the infection of oats by F. langsethiae. Fusarium langsethiae DNA was quantified in significantly larger amounts $(\mathrm{p}<0.05)$ in minimum tilled with incorporated straw plot samples than in other plot samples. It was also shown that inoculated straw had no significant effect $(p>0.05)$ on oat infection by $F$. langsethiae as quantified by DNA concentration. HT-2+T-2 quantification and analysis, gave no good evidence that either inoculation or cultural practice had any significant influence on the concentration of mycotoxins in the samples $(\mathrm{p}>0.05)$, but samples from minimum tillage with incorporated straw plots resulted in 2.5 times more HT-2+T-2 toxins than samples from ploughed with removed straw. These findings indicate the importance of tillage and crop debris management in the mitigation in an effort to prevent $F$. langsethiae infection, colonization and possible contamination of oats with HT-2 and T-2 toxins.
\end{abstract}

Keywords: Fusarium langsethiae, HT-2 and T-2 toxins, oats, small-cereal grains, agronomy.

Fusarium langsethiae has been isolated from infected small-grain cereals in a number of European countries, where also, high levels HT-2 and T-2 toxins have been reported to be associated (Infantino et al., 2004; Imathiu et al., 2013a; Morcia et al., 2016). In regard to these toxins production, F. langsethiae can be considered a food and feed safety hazard if found to be contaminating the raw materials with these toxins. The fungus has been shown to produce high amounts of HT-2 and T-2 toxins in small-grain cereals, particularly in oats (Imathiu et al., 2013b; Dedeurwaerder et al., 2014). T-2 and HT-2 (a deacetylated form of T-2) are type A trichothecenes which are usually found together in contaminated cereal grains and their products. High levels of these mycotoxins have been reported in warm and dry summers in Norway (Langseth and Rundberget,

* Corresponding author; e-mail: samuel.imathiu@jkuat.ac.ke 
1999), although the actual epidemiology of the fungus is not well known (Imathiu at al., 2013a). Recently, the European Commission (EC) has been considering legislation of HT-2 and T-2 toxins. Legislative limits for the sum of HT-2 and T-2 toxins are to be set by the EC after the generation of more occurrence data, development of sensitive analytical methods and after more research has been carried out on the factors involved in the presence of these toxins in cereal and cereal-based products (Commission Regulation Number 1881/2006) (EC, 2006). Indicative levels for the sum of HT-2 and T-2 toxins in cereals and cereal products have recently been issued by the EC in the Recommendation 2013/165/EC (EC, 2013).

HT-2 and T-2 toxins, two of the most toxic trichothecenes are powerful inhibitors of protein synthesis, often cytotoxic, causing skin and mucosa erosions and reduction of lymphocytes, immune defence and growth in exposed animals (Pettersson et al., 2010). In European cereal production, $F$. langsethiae has been implicated as the main producer of HT-2 and T-2 trichothecenes (Edwards et al., 2012).

The significance of $F$. langsethiae on fusarium head blight (FHB) (fusarium panicle blight (FPB) in the case of oats) complex is not known. Although it has occasionally been isolated in association with head blight pathogens, it has not been shown to cause the disease symptoms on cereals under both controlled and field experiments (Imathiu et al., 2013a; Imathiu et al., 2013b; Opoku et al., 2013; Morcia et al., 2016). Fusarium head blight, also known as scab or head fusariosis describes a disease of small-grain cereals that include oats. This disease is caused by several species of the genus Fusarium and Microdochium nivale (Miller, 1994; Parry et al., 1995). Whether or not the agronomic or cultural field practices affects infection of small-grain cereals with $F$. langsethiae and subsequent production of HT-2 and T-2 toxins on the crop, as demonstrated for the FHB pathogens is not well understood. Fusarium head blight epidemics are generally considered to originate from inoculum associated with stubble and straw, particularly those from smallgrain cereals and maize, which means that practices such as crop debris management, and land preparation methods such as tillage techniques are likely to influence fungal infection (Pirgozliev et al., 2003; Champeil et al., 2004b; Maiorano et al., 2008). This study was designed to investigate the effect of agronomic/cultural practices as well as the effect of inoculated oats straw on the infection and mycotoxin production by $F$. langsethiae on oats cultivar Gerald.

\section{Materials and Methods}

\section{Collection and preparation of oat straw for artificial inoculum}

Dry oat straw from the previous field trial (2005/2006) was shredded to approximately $6 \mathrm{~cm}$ long pieces using a garden shredder (AL-KO, UK). Shredded straw was autoclaved (1 kg per bag with $500 \mathrm{ml}$ added distilled water) at $121{ }^{\circ} \mathrm{C}$ for $1 \mathrm{~h}$ and cooled to room temperature. Some shredded straw was not autoclaved and was used as a control treatment. 


\section{Preparation of inoculum broth culture}

Fusarium langsethiae isolates used (Table 1) were grown on potato dextrose agar (PDA) separately at room temperature for 10 days. Each isolate colony on PDA was cut into small pieces with a sterile scalpel and inoculated into sterile $200 \mathrm{ml}$ malt extract broth (Merck, Germany) in a $1 \mathrm{~L}$ conical flask which was then plugged with cotton wool and sealed with aluminium foil. Inoculum was incubated at room temperature under natural light for 11 days after which all $1 \mathrm{~L}$ isolate inoculum broths were mixed together to produce a composite inoculum in a sterile container.

\section{Table 1}

Fusarium langsethiae oat isolates used for inoculating oat straw

\begin{tabular}{lcc}
\hline Isolate & Host source & Mycelium colour \\
\hline$F l 2004 / 37 / 1$ & Oat & Orange \\
$F l 2004 / 57$ & Oat & Orange \\
$F l 2004 / 31$ & Oat & White \\
$F l 026 / 1$ & Oat & Orange \\
$F l 2004 / 9$ & Oat & White \\
$F l 062 / 2$ & Oat & White \\
$F l 077 / 03$ & Oat & White \\
$F l 2004 / 59 / 1$ & Oat & White \\
$F l 2004 / 37 / 2$ & Oat & Orange \\
$F l 062 / 1$ & Oat & White \\
$F l 2004 / 59$ & Oat & Purple \\
\hline
\end{tabular}

\section{Inoculation of sterilized shredded oat straw}

A $350 \mathrm{ml}$ of mixed inoculum broth was used to inoculate each of $1 \mathrm{~kg}$ of sterilized oat straw. To each $1 \mathrm{~kg}$ of inoculated oat straw, $500 \mathrm{ml}$ of sterilized malt extract broth was added to promote growth and proliferation of the culture and then incubated at room temperature under natural light. The inoculum was mixed once every 2 days and was ready for application after 47 days. Some shredded autoclaved straw was not inoculated and was used as a control treatment.

\section{Field set-up and treatment application}

Oat cultivar Gerald was drilled at a rate of 275 seeds $\mathrm{m}^{-2}$ in a field whose previous crop was wheat. The trial was made up of sixteen $24 \times 24$ m main plots consisting of randomised minimum tillage and plough with and without straw incorporation. Accord Plough and Press Combination Drill (Dowdeswell, Germany) and Vaderstad Light Cultivation and Direct Drill (Smarag-Lemken, Germany) were used for plough and minimum 
tillage, respectively. The four levels under minimum tillage and plough were; minimum tilled with straw removed, minimum tilled with incorporated straw, ploughed with straw removed, and ploughed with incorporated forecrop straw. Four small subplots $(2 \times 2 \mathrm{~m})$ were laid within each main plot and constituted experimental units (64 in total) in which the treatments were applied after randomisation within each main plot. The four subplots were equidistant from each other. Four inoculation levels were used, i.e. F. langsethiae inoculated oat straw (840 g), autoclaved oat straw (470 g), untreated straw (312 g) and non-inoculated controls. Weight of straw applied, depending on treatment was different because of addition of broth culture and sterilized broth to some (e.g. F. langsethiae-inoculated straw) but not to others (e.g. just sterilized straw). Treatments were applied by hand broadcast when the crop flag leaves had fully emerged, Zadoks growth stage (GS) 39 (Zadoks et al., 1974). The trial was maintained according to Harper Adams University's standard crop husbandry practices. A temperature data logger was used to record the minimum and maximum temperatures during the study period. The amount of rainfall and relative humidity during the same study period was also recorded.

\section{Disease development monitoring}

Disease monitoring by visual assessment of FPB symptoms was started 5 days post-inoculation and once every week until after panicle tissues senesced (Zadoks GS 88-90). Symptoms characteristic of FPB development monitored included pink discolouration at the base of the spikelets and partial prematurely bleached spikelets (Wong et al., 1995; Chongo et al., 2001).

\section{Harvesting and postharvest sample analyses}

Each subplot was harvested at grain maturity (Zadoks GS 92) using a Seedmaster Plot combine harvester (Wintersteigher, Ried im Innkreis, Austria). Samples were weighed and dried to a moisture content of $13 \%$. Each sample was divided into two representative samples by a ripple grain divider (Novaliter Laboratories, UK) before half of it was milled (ZM100, Retsch, Germany), mixed using a tumbler mixer (Lab-mixer, Pascall, UK), packaged in poly-grip bags and frozen at $-20^{\circ} \mathrm{C}$ for subsequent $F$. langsethiae DNA and HT-2 and T-2 mycotoxin extraction and quantification.

\section{DNA extraction and $\mathrm{F}$. langsethiae DNA quantification by real-time PCR}

Milled samples were thoroughly mixed using a tumbler mixer (Lab-mixer, Pascall, UK). Six gram sub-samples were weighed in $50 \mathrm{ml}$ centrifuge tubes and DNA extracted using CTAB buffer. Thirty millilitres of CTAB buffer was added to each tube, mixed and incubated at $65{ }^{\circ} \mathrm{C}$ for $1 \mathrm{~h}$. Tube contents were shaken by hand and centrifuged at $3,000 \times g$ for $15 \mathrm{~min}$. A $0.9 \mathrm{ml}$ volume of supernatant was removed and added to $0.3 \mathrm{ml}$ potassium acetate $(5 \mathrm{M})$ in a sterile $1.9 \mathrm{ml}$ Eppendorf tube, mixed for $1 \mathrm{~min}$ and frozen for $1 \mathrm{~h}$ at $-20^{\circ} \mathrm{C}$. Tube contents were thawed at room temperature, $0.6 \mathrm{ml}$ chloroform added, mixed for $1 \mathrm{~min}$ and centrifuged at $12,000 \times g$ for $15 \mathrm{~min}$. One millilitre of the aqueous 
phase was removed from each tube and transferred to a fresh $1.9 \mathrm{ml}$ Eppendorf tube containing $0.8 \mathrm{ml}$ of $100 \%$ isopropanol and mixed for 1 min before centrifuging at $12,000 \times g$ for $15 \mathrm{~min}$. The resulting DNA pellets were washed twice with $1 \mathrm{ml} 44 \%$ isopropanol. Pellets were air dried before re-suspending in $0.2 \mathrm{ml}$ TE buffer and incubating at $65^{\circ} \mathrm{C}$ for $25 \mathrm{~min}$. Tube contents were vortexed and left at room temperature overnight before spinning at $12,000 \times g$ for $5 \mathrm{~min}$. All extracts were stored at $-20^{\circ} \mathrm{C}$. The quantification of F. langsethiae biomass was carried out using the methodology described by Edwards et al. (2012).

\section{Determination of HT-2 and T-2 concentration in oat samples}

The analysis of combined HT-2 and T- 2 toxins was carried out by an ELISA methodology as detailed by Edwards et al. (2012). Currently, there are no commercial ELISA assays which quantify HT-2 and T-2 together. Commercially available assays for T-2 partly cross-react with HT-2. The concentration of combined HT-2 and T-2 was estimated based on the known ratio that the two related toxins occur together in cereals (Edwards, 2007) and the known cross-reactivity of a T-2 ELISA assay with HT-2. HT-2 and T-2 trichothecene concentration in milled samples was estimated using Ridascreen ${ }^{\circledR}$ Fast T-2 Toxin enzyme-linked immunosorbent assay (R-Biopharm AG, Darmstadt, Germany). Five grams of milled sample was weighed in $50 \mathrm{ml}$ centrifuge tubes after which $25 \mathrm{ml}$ of $70 \%$ methanol was added. Tubes were vigorously shaken for $3 \mathrm{~min}$ and centrifuged at $3,000 \times g$ for $5 \mathrm{~min}$. One millilitre of the resulting supernatant was diluted with $1 \mathrm{ml}$ of distilled water and, $50 \mu 1$ of the resulting mixture was used per well in the test according to the manufacturer's instructions.

Combined HT-2 and T-2 concentration was estimated based on the following relationships: (1) ratio of HT-2 to T-2 was determined to be 3.3:1 in oat samples (Edwards, 2007), (2) HT- 2 cross-reacts with Ridascreen ${ }^{\circledR}$ Fast T- 2 ELISA antibody $11 \%$ compared to T-2, consequently the estimate of combined HT-2 and T-2 was determined from the T-2 Fast ELISA value (X) by the equation:

$$
\mathrm{HT}-2+\mathrm{T}-2=(\mathrm{X} / 1.362)+(\mathrm{X} / 1.362) / 0.303
$$

\section{Data analysis}

Analysis of all data was performed using GenStat (Tenth Edition, Rothamsted Experimental Station, UK). Mean separation was carried out using Tukey's test $(\mathrm{P}<0.05)$.

\section{Results}

The minimum and maximum temperatures were highly variable during the period after artificial inoculum application, i.e. day 35 (Fig. 1). The ranges were 0 to $16^{\circ} \mathrm{C}$ and 10 to $26^{\circ} \mathrm{C}$ for minimum and maximum temperature, respectively. Rainfall was generally experienced continuously for 11 days post-inoculation but in relatively low amounts (Fig. 


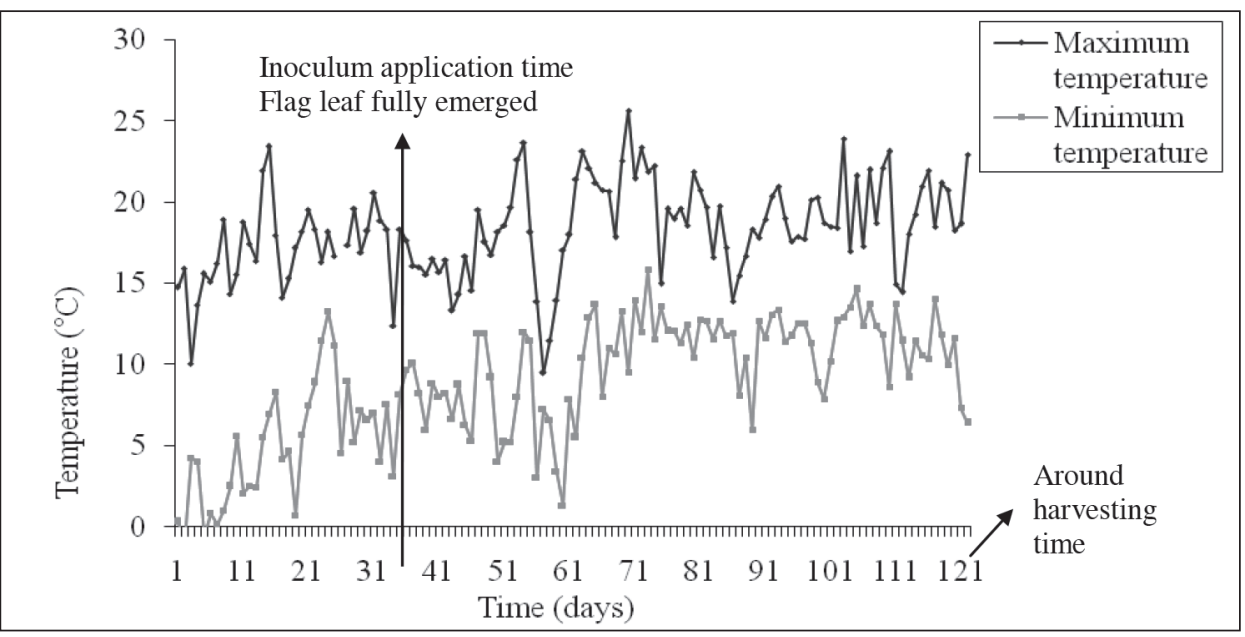

Fig. 1. Minimum and maximum temperature pattern during the study period

2). The highest amount of rainfall recorded was $42 \mathrm{~mm}$ on day nine post-inoculation after $50-100 \%$ of panicles had emerged (Zadoks GS 55-59). Rainfall was variable post-inoculation, while humidity was relatively high especially after eight days after inoculum application, averaging above $80 \%$.

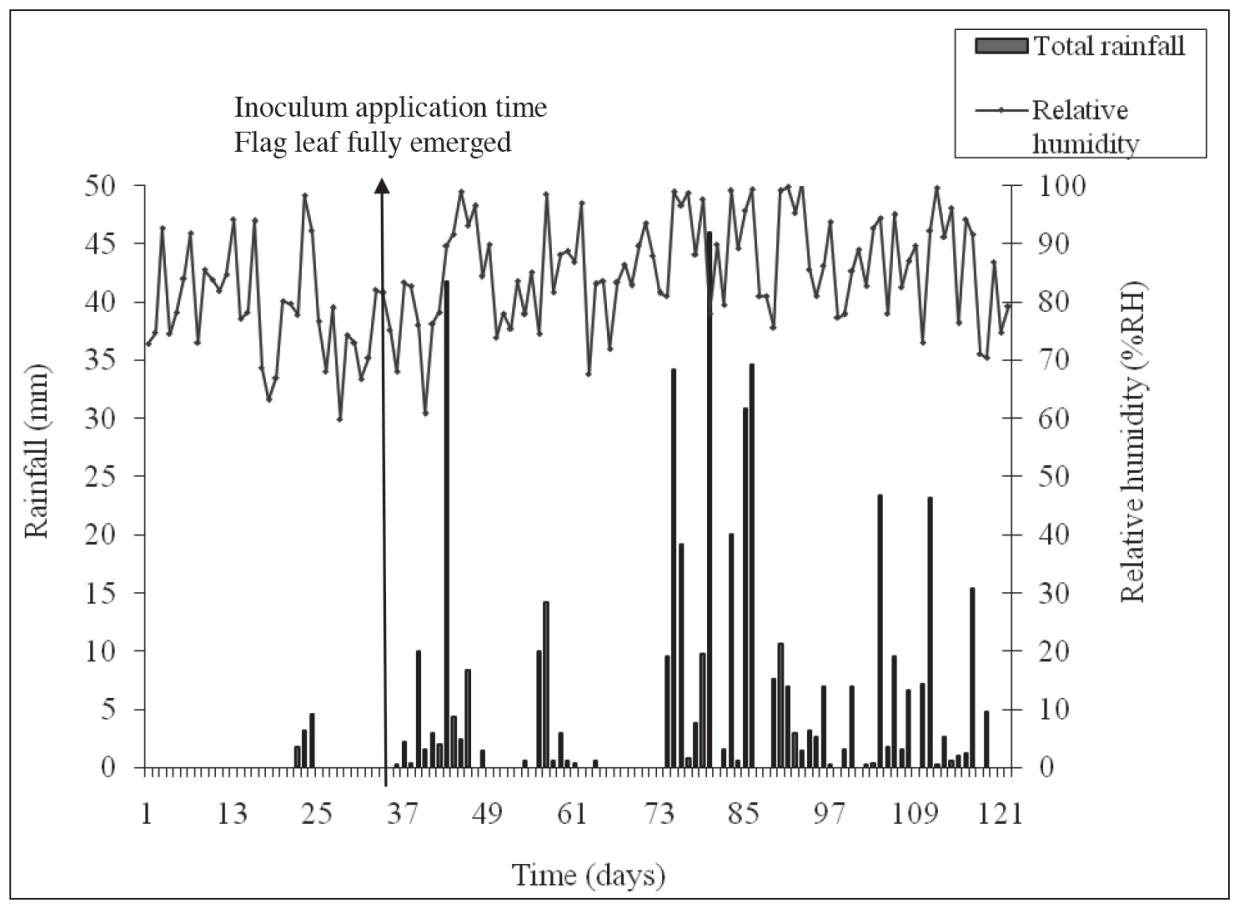

Fig. 2. Rainfall and relative humidity experienced during the study period 
Disease symptoms characteristic of FPB of oats were observed in a few panicles particularly in minimum tilled with incorporated straw and minimum tilled with straw removed main plots. In the most diseased panicles, only a maximum of three spikelets per panicle were observed exhibiting this kind of symptom. The main plots where these few symptoms were found were characterised by growth of a lot of weeds and some insects in addition to plant debris. Fusarium graminearum was the only fungus isolated from panicles exhibiting FPB disease symptoms, while $F$. langsethiae was not recovered from any of the diseased spikelets. Ploughed plots plant density ranged from 181-222 plants $\mathrm{m}^{-2}$ while minimum tilled plots plant density ranged from 16-93 plants $\mathrm{m}^{-2}$.

An ANOVA revealed that there was no significant interaction effect between inoculation and agronomic/cultural practice. It also showed that inoculated straw had no effect on oat infection by $F$. langsethiae as quantified by DNA concentration $(\mathrm{P}>0.05)$ (Table 2). However, cultural field practices clearly had effects on the infection of oats by F. langsethiae. Fusarium langsethiae DNA was quantified in significantly larger amounts $(\mathrm{P}<0.05)$ in minimum tilled with incorporated straw plot samples than in any other agronomic/cultural practice. For all other cultural practices, their F. langsethiae DNA concentration did not differ significantly from each other. Minimum tilled with incorporated straw samples gave three times more $F$. langsethiae DNA than minimum tilled with straw removed, and four times more than ploughed with straw removed and ploughed with incorporated straw samples. Fusarium langsethiae DNA concentration, which was used as a measure of fungal infection ranged from $0.0015 \mathrm{pg} \mathrm{ng}^{-1}$ (minimum tilled with straw removed) to $0.019 \mathrm{pg} \mathrm{ng}^{-1}$ (minimum tilled with incorporated straw). Although there were no significant differences among the latter three cultural practices, the mean values of the F. langsethiae biomass would suggest that there may have been less infection in ploughed than in minimum tilled plots.

Table 2

Fusarium langsethiae DNA concentration in oat variety Gerald at harvest

\begin{tabular}{|c|c|c|c|c|c|}
\hline \multirow[b]{2}{*}{ Agronomy } & \multicolumn{5}{|c|}{ F. langsethiae DNA $\left(\mathrm{pg} \mathrm{ng}^{-1}\right)$} \\
\hline & $\begin{array}{l}\text { Autoclaved } \\
\text { straw }\end{array}$ & $\begin{array}{l}\text { Inoculated } \\
\text { straw }\end{array}$ & No straw & $\begin{array}{l}\text { Untreated } \\
\text { straw }\end{array}$ & $\begin{array}{c}\text { Factor mean } \\
\text { (agronomy) }\end{array}$ \\
\hline Minimum tilled with straw removed & 0.012 & 0.0015 & 0.0030 & 0.0018 & $0.0045^{\mathrm{a}}$ \\
\hline Minimum tilled with incorporated straw & 0.016 & 0.019 & 0.015 & 0.0064 & $0.0140^{\mathrm{b}}$ \\
\hline Ploughed with straw removed & 0.0032 & 0.0016 & 0.0039 & 0.0059 & $0.0036^{\mathrm{a}}$ \\
\hline Ploughed with incorporated straw & 0.0034 & 0.0024 & 0.0064 & 0.0018 & $0.0035^{\mathrm{a}}$ \\
\hline Factor mean (inoculation) & 0.0085 & 0.0061 & 0.0071 & 0.0040 & \\
\hline P-value & \multicolumn{2}{|c|}{ Inoculation } & Agronomy & \multicolumn{2}{|c|}{ Inoculation $\times$ Agronomy } \\
\hline $\operatorname{LSD}(5 \%)$ & \multicolumn{2}{|c|}{0.267} & 0.006 & \multicolumn{2}{|c|}{0.207} \\
\hline DF & \multicolumn{2}{|c|}{-} & $\begin{array}{c}0.006 \\
36\end{array}$ & \multicolumn{2}{|c|}{-} \\
\hline
\end{tabular}

Values are means of 16 replicates and different superscript letters indicate statistically significant differences in F. langsethiae DNA based on Tukey's test $(\mathrm{P}<0.05)$ 
HT-2 + T-2 toxin quantification and analysis, gave no good evidence that either inoculation or cultural practice had any significant influence on the concentration of these mycotoxins in the samples $(\mathrm{P}>0.05)$ (Table 3). Although there were no significant differences among cultural practices in HT-2 + T-2 toxin concentration, the trend appeared similar to the F. langsethiae DNA concentration detailed in (Table 2). Samples from minimum tillage with incorporated straw plots resulted in 2.5 times more HT-2 + T-2 toxin concentration than samples from ploughed with removed straw. HT-2 + T-2 toxin concentration ranged from $156 \mathrm{ppb}$ (ploughed with straw removed) to $840 \mathrm{ppb}$ (minimum tilled with incorporated straw).

Table 3

Transformed $\left(\log _{10}\right)$ HT-2 + T-2 mycotoxin content in oat variety Gerald samples at harvest

\begin{tabular}{lccccc}
\hline & \multicolumn{4}{c}{ HT-2+T-2 (ppb) } \\
\cline { 2 - 6 } Agronomy & $\begin{array}{c}\text { Autoclaved } \\
\text { straw }\end{array}$ & $\begin{array}{c}\text { Inoculated } \\
\text { straw }\end{array}$ & No straw & $\begin{array}{c}\text { Untreated } \\
\text { straw }\end{array}$ & $\begin{array}{c}\text { Factor mean } \\
\text { (agronomy) }\end{array}$ \\
\hline Minimum tilled with removed straw & $2.389(254)$ & $2.419(269)$ & $2.297(204)$ & $2.255(187)$ & $2.34(229)$ \\
Minimum tilled with incorporated straw & $2.629(475)$ & $2.400(259)$ & $2.674(840)$ & $2.434(327)$ & $2.534(475)$ \\
Ploughed with straw removed & $2.246(179)$ & $2.153(180)$ & $2.378(247)$ & $2.169(156)$ & $2.237(190)$ \\
Ploughed with incorporated straw & $2.192(174)$ & $2.350(267)$ & $2.184(180)$ & $2.350(239)$ & $2.969(215)$ \\
Factor mean (inoculation) & $2.364(271)$ & $2.33(244)$ & $2.383(368)$ & $2.302(227)$ \\
P-value & Inoculation & Agronomy & Inoculation $\times$ Agronomy \\
DF & 0.59 & 0.184 & 0.147 \\
& & 35 & & \\
\hline
\end{tabular}

Values are means of 16 replicates. Untransformed values in parenthesis

\section{Discussion}

Conducting the trial in the field enabled infection and disease symptoms resulting from the treatments to be observed under natural conditions. The study demonstrated that F. langsethiae, although it could be part of FHB complex, did not result in typical FPB disease symptoms in the infected oat panicles. Rarely observed FPB symptoms have been reported in oats in the field caused by the most pathogenic and aggressive Fusarium species including $F$. avenaceum, $F$. culmorum and $F$. graminearum although the incidence and severity in the crop has been observed to be significantly lower compared with wheat, in previous reported studies (Gilbert and Tekauz, 2000). Fusarium panicle blight symptoms observed in this study were caused by $F$. graminearum which was the only FPB pathogen isolated from the diseased spikelets and whose inoculum in the crop may have come from the crop debris of the preceding wheat crop.

Symptoms have been used to quantify FHB diseases during natural infection or when small cereal heads are sprayed with artificial inoculum. This is probably because of their relative speed and ease of visual assessments of FHB (mainly done in wheat) which is easily recognised in the field and provides the earliest information for decision 
making prior to maturity and harvesting of the crop (Shaner, 2003). Where reliance on field disease symptoms is depended on, this study has demonstrated that it may overlook symptomless infections caused by $F$. langsethiae in oats. The reason for $F$. langsethiae not being able to cause head blight of oats is not known but may be because it is a weak pathogen as weak pathogens have been reported to cause little or no disease symptoms on cereals, particularly in oats which is reported to be more resistant to FHB compared with wheat (Clear et al., 1996; Langseth and Elen, 1996; Langseth and Stabbetorp, 1996; Liu et al., 1997; Tekauz et al., 2004). According to Henriksen and Elen (2005), infection of oats by less pathogenic species of Fusarium may not result in typical FPB symptoms. This statement is supported by that of Osborne and Stein (2007) who have reported the inability of some Fusarium species including F. poae and F. sporotrichioides, considered weak pathogens, to cause disease symptoms in cereals upon infection. Apparently, F. langsethiae may be behaving as a symptomless endophyte upon infection. The most aggressive Fusarium species known to cause significant visual symptoms of FHB in wheat but rarely in oats are $F$. culmorum and $F$. graminearum; therefore, if $F$. langsethiae is less pathogenic than these two, which it seems to be, it is less likely to cause noticeable FPB symptoms in oats. Recently, a number of researchers including Imathiu et al. (2013a and 2013b); Opoku et al. (2013) and Morcia et al. (2016) have reported asymptomless infection by F. langsethie on oats, wheat and barley. Symptomless infections on these cereals caused by $F$. langsethiae as demonstrated in these studies calls for novel measures of infection monitoring to be designed and implemented if correct diagnosis is to be made in the field and mitigation interventions implemented in good time.

The study has reported low infection levels on oats by F. langsethiae as measured by the amount of DNA concentration in the harvested samples. Oats are generally considered less prone to FHB pathogens than wheat as they are reported to be more resistant than wheat (Gilbert and Tekauz, 2000) which may explain the low level of infection. Fusarium culmorum and $F$. graminearum have also been reported to be aggressive pathogens of wheat and to some extent oats (Parry et al., 1994; Parry et al., 1995; Gilbert and Tekauz, 2000) while the aggressiveness of $F$. langsethiae on cereals has never been reported.

The importance of cultural field practices was evident in this study where significant differences were observed among the four agronomic practices. Many authors have reported on the importance of cultivation methods and the contribution of crop residue to various plant diseases, particularly in FHB of wheat (Parry et al., 1995; Champeil et al., 2004a; Champeil et al., 2004b). Presence of crop debris on the soil surface has been shown to increase the incidence and severity of FHB of wheat, the disease intensity depending on the type of crop debris, the pathogen involved, whether or not the field was ploughed and the weather conditions. This study has shown that, infection of oats can be affected in the same way as infection of wheat by cultural field practices. Minimum tilled plots with straw incorporated had significantly more F. langsethiae DNA in harvested oat grains than in the other three cultural field practices (minimum tillage with straw removed, ploughed with straw removed and ploughed with straw incorporated) suggesting greater infection in the former. The differences in the cultural practices in this study can be mainly attributed to the method of cultivation. Crop residue can be a source of inoculum for FPB where it can act as a substrate on which the pathogens can 
survive as saprophytes and be able to infect the crop at its most susceptible stage, the flowering stage (Xu, 2003).

The way the field is managed determines whether the natural inoculum present in the residue is available for infection or not. Ploughing served to bury the residue, significantly reducing the amount of inoculum available for causing an infection. Minimum tillage on the other hand would result to a considerable amount of debris lying on the soil, which, if it contains natural inoculum may cause greater infection. The results reported from this study support those of Maiorano et al. (2008) who reported increased infection by FHB pathogens as a result of direct drilling or minimum tillage in a wheat field as well as those of Miller et al. (1998) and Pirgozliev et al. (2003) who reported that ploughing is one of the most effective previous crop residue management strategies as it removes plant debris from the soil surface and buries it reducing the amount of inoculum available for FHB infection of wheat. These results also agree with those of Parikka et al. (2007) who reported high levels of $F$. langsethiae infection in oats in direct-drilled plots compared with ploughed plots.

In addition to making natural inoculum available for infection, minimum tillage may also result in a higher weed population. It was observed in this study that minimum tilled plots had more weeds, both broad-leaved and grasses and that these plots were infested with more insects than ploughed plots. These weeds might have acted as an alternative source of $F$. langsethiae inoculum and insects may have acted as vectors transferring the inoculum to the oat panicles. Jenkinson and Parry (1994) reported isolating $F$ culmorum and $F$. graminearum from a wide range of weeds in the UK indicating that weeds could be a source of Fusarium inoculum. The fact that several Fusarium species have been isolated on a wide range of insects indicates that insects may also act as vectors (Miller et al., 1998). For example, mites have been reported to transport $F$. poae spores (Parry et al., 1995), and according to Gordon (1959), insects have been reported to carry Fusarium species including $F$. poae, $F$. avenaceum and F. culmorum.

Minimum tilled plots also had a poor crop stand compared with the ploughed plots which might have come about as a result of poor drilling as the ground in these plots was not ideal as a result of shallow cultivation and a lot of crop debris. This might have reduced the canopy effect in the splash dispersal of fungal conidia as the rainfall-splashed natural inoculum conidia from the soil surface/debris or plant leaves to the oat panicles was not restricted as much as would be expected where plants were densely populated as in the ploughed plots. This might also have contributed in the level of infection by F. langsethiae in minimum tilled plots. The true effect of the method of cultivation in this study may not be apparent owing to the effect, for example, minimum tillage had on plant population. Plant population has been reported to influence the infection of wheat by FHB pathogens (Champeil et al., 2004a).

Results obtained after mycotoxin analysis from the study showed that neither inoculation nor agronomical/cultural practice influenced HT-2 + T-2 toxins significantly. However, a trend of HT-2 + T-2 toxin contamination level was evident which depended on method of cultivation employed. The trends these toxins concentration were similar to that reported in this investigation concerning infection as determined by $F$. langsethiae DNA concentration. Oat grains from ploughed plots were less contaminated with HT-2 + T-2 
toxin compared with those from minimum tilled plots, an observation that agrees with that of Parikka et al. (2007) who reported higher concentrations of these mycotoxins in oat grains harvested from direct-drilled plots than from ploughed plots. Edwards (2007) also reported lower HT-2 + T-2 toxin in oat samples from ploughed fields than those which were not ploughed which suggested that crop debris was important in the epidemiology of the HT-2 and T-2-producing Fusarium species. It appears that factors predisposing oats to infection by $F$. langsethiae also influence HT-2 and T-2 mycotoxin contamination in the grains in the same way, and that the higher the level of infection, the higher the likelihood of quantifying high levels of these mycotoxins in the grains. A naturally infected commercial oat crop has been reported to accumulate high HT-2 and T-2, with a median, mean and maximum of 213, 570 and 9,990 ppb (Edwards, 2007).

\section{Acknowledgements}

Authors wish to thank Quaker Oats, Home-Grown Cereals Authority and Harper Adams University for funding this study.

\section{Literature}

Champeil, A., Dore, T. and Fourbet, J. F. (2004a): Fusarium head blight: epidemiological origin of the effects of cultural practices on head blight attacks and the production of mycotoxins by Fusarium in wheat grains. Plant Sci. 166, 1389-1415.

Champeil, A., Fourbet, J. F., Dore, T. and Rossignol, L. (2004b): Influence of cropping system on fusarium head blight and mycotoxin levels in winter wheat. Crop Protect. 23, 531-537.

Chongo, G., Gossen, B. D., Kutcher, H. R., Gilbert, J., Turkington, T. K., Fernandez, M. R. and McLaren, D. (2001): Reaction of seedling roots of 14 crop species to Fusarium graminearum from wheat heads. Can. J. Plant Pathol. 23, 132-137.

Clear, R. M., Patrick, S. K., Nowicki, T., Gaba, D., Edney, M. and Babb, J. C. (1996): The effect of hull removal and pearling on Fusarium species and trichothecenes in hulless barley. Can. J. Plant Sci. 77, 161-166.

Dedeurwaerder, G., Ghysselinckx, J., Hellin, P., Janssen, F., Duvivier, M. and Legrève, A. (2014): Detection of Fusarium langsethiae on wheat in Belgium. Eur. J. Plant Pathol. 139, 453-455.

Edwards, S. G. (2007): Investigation of fusarium mycotoxins in UK barley and oat production. HGCA Project Report No. 415. HGCA, London.

Edwards, S. G., Imathiu, S. M., Ray, R. V., Back, M. and Hare, M. C. (2012): Molecular studies to identify the Fusarium species responsible for HT-2 and T-2 mycotoxins in UK oats. Int. J. Food Microbiol. 156, $168-175$.

European Commission (2006): Commission regulation (EC) No 1881/2006 of 19 December 2006 setting maximum levels of certain contaminants in foodstuffs. Off. J. Eur. Communities: Legis. http://eur-lex.europa. eu/LexUriServ/LexUriServ.do?uri=OJ:L:2006:364:0005:0024:EN:PDF. Accessed on 26/11/2016.

European Commission (2013): Commission recommendation (2013/165/EU) of 27 March 2013 on the presence of T-2 and HT-2 toxin in cereals and cereal products. Off. J. Eur. Communities: Legis. http://eur-lex.europa.eu/legal-content/EN/ALL/?uri=CELEX\%3A32013H0165. Accessed on 26/11/2016.

Gilbert, J. and Tekauz, A. (2000): Review: recent developments in research on fusarium head blight of wheat in Canada. Can. J. Plant Pathol. 22, 1-8.

Gordon, W. L. (1959): The occurrence of Fusarium species in Canada IV. Taxonomy and geographic distribution of Fusarium species on plants, insects and fungi. Can. J. Botany. 37, 257-290.

Henriksen, B. and Elen, O. (2005): Natural Fusarium grain infection level in wheat, barley and oat after early application of fungicides and herbicides. J. Phytopathol. 153, 214-220. 
Imathiu, S. M., Edwards, S. G., Ray, R. V. and Back, M. (2013a): Fusarium langsethiae - a HT-2 and T-2 toxins producer that needs more attention. J. Phytopathol. 161, 1-10.

Imathiu, S. M., Ray, R. V., Back, M., Hare, M. C. and Edwards, S. G. (2013b): A survey investigating the infection of Fusarium langsethiae and production of HT-2 and T-2 mycotoxins in UK oat fields. J. Phytopathol. 161, 553-561.

Infantino, A., Santori, A., Aureli, G., Belocchi, A., De Felice, S., Tizzani, L., Lattanzio, V. M. T., Haidukowski, M. and Pascale, M. (2004): Occurrence of Fusarium langsethiae strains isolated from durum wheat in Italy. J. Phytopathol. 163, 612-619.

Jenkinson, P. and Parry, D. W. (1994): Isolation of Fusarium species from common broad-leaved weeds and their pathogenicity to winter wheat. Mycol. Res. 98, 776-780.

Langseth, W. and Elen, O. (1996): Differences between barley, oats and wheat in the occurrence of deoxynivalenol and other trichothecenes in Norwegian grain. J. Phytopathol. 144, 113-118.

Langseth, W. and Stabbetorp, H. (1996): The effect of lodging and time of harvest on deoxynivalenol contamination in barley and oats. J. Phytopathol. 144, 241-245.

Langseth, W. and Rundberget, T. (1999): The occurrence of HT-2 toxin and other trichothecenes in Norwegian cereals. Mycopathologia 147, 157-165.

Liu, W. Z., Langseth, W., Skinnes, H., Elen, O. N. and Sundheim, L. (1997). Comparison of visual head blight ratings, seed infection levels and deoxynivalenol production for assessment of resistance in cereals inoculated with Fusarium culmorum. Eur. J. Plant Pathol. 103, 589-595.

Maiorano, A., Blandino, M., Reyneri, A. and Vanara, F. (2008): Effect of maize residues on the Fusarium spp. Infection and deoxynivalenol (DON) contamination of wheat grain. Crop Protect. 27, 182-188.

Miller, J. D. (1994): Epidemiology of fusarium ear diseases of cereals. In: J. D. Muller and H. L. Trenholm (eds): Mycotoxins in Grains. Compounds Other Than Aflatoxin. Eagan Press, St. Paul, Minn. pp. 19-36.

Miller, J. D., Culley, J., Fraser, K., Hubbard, S., Meloche, F., Ouellet, T., Seaman, W. L., Seifert, K. S., Turkington, K. and Voldeng, H. (1998): Effect of tillage practice on fusarium head blight of wheat. Can. J. Plant Pathol. 20, 95-103.

Morcia, C., Tumino, G., Ghizzoni, R., Badeck, F. W., Lattanzio, V. M. T., Pascale, M. and Terzi, V. (2016): Occurrence of Fusarium langsethiae and T-2 and HT-2 toxins in Italian malting barley. Toxins 8, 247.

Opoku, N., Back, M. and Edwards, S. G. (2013): Development of Fusarium langsethiae in commercial cereal production. Eur. J. Plant Pathol. 36, 159-170.

Osborne, L. E. and Stein, J. M. (2007): Epidemiology of fusarium head blight of small-grain cereals. Int. J. Food Microbiol. 119, 103-108.

Parikka, P., Hietaniemi, V., Ramo, S. and Jalli, H. (2007): The effect of cultivation practices on Fusarium langsethiae infection of oats and barley. Fusarium diseases in cereals - potential impact from sustainable cropping systems. Proc. of the COST SUSVAR Fusarium Workshop, Vogelsang. Velence, Hungary (0102 June 2007).

Parry, D. W., Jenkinson, P. and Lees, A. K. (1994): The cereal Fusarium complex. In: J. P. Blakeman and B. Williamson (eds): Ecology of Plant Pathogens. CAB International, Wellingford, UK. pp. 301-320.

Parry, D. W., Jenkinson, P. and McLead, L. (1995): Fusarium ear blight (scab) in small grain cereals-a review. Plant Pathol. 44, 207-238.

Pettersson, H., Brown, C., Hauk, J., Hoth, S., Meyer, J. and Wessels, D. (2010): Survey of T-2 and HT-2 toxins by LC-MS/MS in oats and oat products from European oat mills in 2005-2009. Food Addit. Contam. B. 4, 110-115.

Pirgozliev, S. R., Edwards, S. G., Hare, M. C. and Jenkinson, P. (2003): Strategies for the control of fusarium head blight in cereals. Eur. J. Plant Pathol. 109, 731-742.

Shaner, G. (2003): Epidemiology of fusarium head blight of small grain cereals in North America. In: K. J. Leonard and W. R. Bushnell (eds): Fusarium Head Blight of Wheat and Barley. American Phytopathol. Soc., St. Paul, Minnesota, USA, pp. 1-87.

Tekauz, A., McCallum, B., Ames, N. and Fetch, J. M. (2004): Fusarium head blight of oats-current status in western Canada. Can. J. Plant Pathol. 26, 473-479. 
Wong, L. S. L., Abramson, D., Tekauz, A., Leisle, D. and McKenzie, R. I. H. (1995): Pathogenicity and mycotoxin production of Fusarium species causing head blight in wheat cultivars varying in resistance. Can. J. Plant Sci. 75, 261-267.

$\mathrm{Xu}, \mathrm{X}$. (2003): Effects of environmental conditions on the development of fusarium ear blight. Eur. J. Plant Pathol. 109, 683-689.

Zadoks, J.C., Chang, T.T. and Kunzak, C.F. (1974): Decimal code for growth stages of cereals. Weed Res. 15, 415-421. 